\title{
Cognitive HF - New Perspectives to Use the High Frequency Band
}

\author{
Invited Paper
}

\author{
Teemu Vanninen, Toni Linden, Matti Raustia \\ Kyynel Oy \\ Oulu, Finland
}

\author{
Harri saarnisaari \\ Centre for Wireless Communications \\ University of Oulu \\ Oulu, Finland
}

\begin{abstract}
The HF band, located in between $3-30 \mathrm{MHz}$, can offer single hop communication channels over a very long distances - even up to around the world. Traditionally, the HF is seen primarily as a solution for long communication ranges although it may also be a perfect choice for much shorter communication ranges when high data rates are not a primary target. It is well known that the HF channel is a demanding environment to operate since it changes rapidly, i.e., channel is available at a moment but the next moment it is not. Therefore, a big problem in HF communications is channel access or channel selection. By choosing the used HF channels wisely, i.e., cognitively, the channel behavior and system reliability considerably improves. This paper discusses about a change of paradigm in HF communication that will take place after applying cognitive principles on the HF system.
\end{abstract}

Keywords- High Frequency, HF, cognitive, software radio

\section{INTRODUCTION}

Radio frequency (RF) transmissions between 3 and $30 \mathrm{MHz}$ are designated in the radio regulations as high frequency (HF) bands. Similarly, symbol "MF" applies to $300-3000 \mathrm{kHz}$ frequencies [1]. Quite often these frequencies overlap in general discussion and the HF band is generally understood from $1.5 \mathrm{MHz}$ to $30 \mathrm{MHz}$, including the upper part of the MF band.

$\mathrm{MF} / \mathrm{HF}$ communications have been used since the early days of $20^{\text {th }}$ century for providing communications via the ionosphere. Advantages of MF/HF communication have been long distance and wide area coverage [1].

The long distance communication in the HF band is possible through skywave propagation, in which radio waves directed at an angle into the sky reflect back to Earth from layers of ionized atoms in the ionosphere. It means that HF radio waves can travel beyond the horizon, around the curve of Earth, i.e, utilization of this transmission form allows to establish long haul communication links [2].

The use of HF band is dominated by the variable nature of the ionospheric propagation so that the operating frequency has to be changed often. There are significant day-to-day changes in ionospheric conditions at the HF band depending on radiation of the Sun and geomagnetic field of Earth.

Suitability of HF frequencies for long range ionospheric communication varies greatly with a complex combination of factors:

- Sunspot cycle

- Season

- Time of the day (sunlight/darkness)

- Solar activity

- $\quad$ Earth's magnetic field

- The upper atmospheric winds

- Geographical location [2].

The radiation from the Sun ionizes the upper atmospheric gases and the resulting free electrons form the ionosphere, which has the property of refracting or reflecting radio waves. In the lower parts of the ionosphere the free electrons have a limited life-time before recombining, and the density of ionization varies approximately with the elevation angle of the sun. The lower parts of the ionosphere also attenuate radio signals. These lower parts of the ionosphere are called the D and $\mathrm{E}$ layers.

Higher in the ionosphere, in the $\mathrm{F}$ layer, electrons have a longer life-time and the ionization density is also strongly affected by winds and by the presence of the Earth's magnetic field [1].

These longer term variations and trends in HF band propagation are predictable on a statistical basis. Many different prediction methods and models are available. Because of statistical approach of propagation models, long-term predictions cannot give a precise estimate of the best frequency to be used at a specific date and time on a specific radio path.

Traditionally, a planned schedule of frequency changes through the day would be prepared for each month. This list of frequencies had been made using statistical propagation predictions and the radio operator would use these frequency 
schedules, together with his experience and actual conditions on the day, and select the best frequency from the limited set of frequencies available to him.

Since late 1970's there's been gaining number of different data transmissions developed for HF band communications. When data was started to be transmitted using HF communications also requirements for an automatic link establishment (ALE) did appear. In 1985 a military standard for the automatic link establishment was accepted. That ALE protocol is called 2G ALE and it is still widely used [1] - [3].

In 1999 3G ALE was accepted as a military standard for faster and more reliable link establishment. The complexity and system requirements have limited the usage of $3 \mathrm{G}$ ALE to military applications and therefore $2 \mathrm{G}$ ALE have still remained as de-facto interoperability standard for the automatic link establishment in HF communications [1] - [4].

Both $2 \mathrm{G}$ and $3 \mathrm{G}$ ALE have a limited number of channels for the link establishment. Typically, 10 to 20 frequency channels are used. These frequencies in so called ALE groups need to be defined manually and changes in propagation conditions can only be taken into account from the statistical point of view.

Combining the hard predictability of HF channels and signals reflecting long distances from ionosphere makes the HF band extremely hard to manage. With traditional frequency management of fixed lists of limited set of frequencies for certain operating hours makes it almost impossible to achieve good communications reliability on the HF band. Therefore, a common assumption of low reliability of HF communications exists. However, these challenges presented for $\mathrm{HF}$ communications makes the HF band an ideal playground for cognitive radio ideas. These will be discussed next.

\section{COGNITIVITY IN BRIEF}

In the area of telecommunications there are multiple definitions for the term "cognitivity". In the simplest form it can be formulated as: "the parameters of a radio are adjusted to fit the current situation in a smart way". This means, basically, two things - the radio itself is aware of the environment and the characteristics of the radio can be adaptively adjusted. Naturally, this also leads to the software radio (SR) concept. A more official definition comes from ITU-R (International Telecommunication Union - radio communication sector) [5], and it is:

"Cognitive radio system (CRS): A radio system employing technology that allows the system to obtain knowledge of its operational and geographical environment, established policies and its internal state; to dynamically and autonomously adjust its operational parameters and protocols according to its obtained knowledge in order to achieve predefined objectives; and to learn from the results obtained."

Next, three acts towards an elementary cognitivity, namely sensing, storing the sensed observations and using the statistics are discussed. As a result, there will be HF radios that can sense the spectrum and use these observations in a smart way to increase the reliability of $\mathrm{HF}$ radio systems using dynamic spectrum access (DSA) techniques.

\section{A. Sensing}

The most obvious sense cognitive radios have is toward the spectrum, i.e. the "eyes" that commit spectrum sensing. The HF spectrum is notorious of its vast variety of different actors, some of which are devices that emit just dull interfering RF energy (artificial noise) and some are HF transmissions on the other side of the planet that the spectrum sensing detects. Therefore, it is easy to anticipate that on the HF spectrum there are almost every imaginable type on signals interfering our own $\mathrm{HF}$ radios and network.

The interfering signals can be roughly divided by their characteristics into three classes. By no means is this an exhaustive list but only to elaborate the division from the viewpoint of practical spectrum sensing.

The classes are:

Static signals These are, for example, broadcast (BC) radio station signals. They are located in well-known frequencies sprinkled along the HF band. Transmitted (TX) power is usually quite high up to $500 \mathrm{~kW}$ [6]. Duration of these transmissions is virtually $24 \mathrm{~h}$ a day, 7 days a week.

Moderately static signals For example, most of the analog or digital voice links belong to this class. The uplink and downlink frequencies are negotiated case by case with, for example, an ALE-protocol after which the frequencies are static unless connection is lost or the connection (link) is finished. Usual data links belong to this class. Telegraphy (morse code) has characteristics of both this class and the next class of Variable signals. Duration of these transmissions is hard to predict; it may vary from tens of seconds to hours.

Variable signals This is the hardest determined class since, basically, all the rest of the HF signal types are in this class. Lighting is one of the strongest RF emitter in this class. Ignition system in older cars and motorcycles causes local short impulses. In some sense the telegraphy belongs to this class since it has impulsive nature. Duration of these signals vary from hundred nano seconds (lighting) [7] to hundreds of milliseconds.

All these signals can be observed in the spectrum depending on the locations of the emitting and the receiving (RX) radio and the skywave (ionospheric) channel conditions.

\section{B. Storing the observation}

While the characteristics of the interfering signals vary a lot, not all of them have relevance when storing the sensed signal statistics. If the history samples of the interfering signal do not predict its future, there is no straightforward use of the statistics of those signals.

In order to define which of the signals our system should or even could take into account, we have to make some initial assumptions. In this paper we'll assume that our HF system utilizes the most state-of-the-art physical (PHY) layer based on the MIL-STD-188-110C standard [8]. 
The 110C standard defines, for example, the following PHY layer parameters:

- $\quad$ Bandwidths: $3 \mathrm{kHz}, 6 \mathrm{kHz}, 9 \mathrm{kHz}, 12 \mathrm{kHz}, 15$ $\mathrm{kHz}, 18 \mathrm{kHz}, 21 \mathrm{kHz}$, and $24 \mathrm{kHz}$

- Symbol rates: 2400, 4800, 7200, 9600, 12000, 14000,16800 and 19200

- Modulations: BPSK, QPSK, 8-PSK, 16-QAM, 32QAM, 64-QAM, 128-QAM, 256-QAM

- Interleaving lengths $110 \mathrm{~ms}-10.24 \mathrm{~s}$ [9].

Thus, the highest channel data rate at $24 \mathrm{kHz}$ channel with 256QAM modulation is $19200 \mathrm{symb} / \mathrm{s} * 8 \mathrm{bits} / \mathrm{symb}=153.6$ kbits/s. From this $120 \mathrm{kbits} / \mathrm{s}$ is user data rate after the synchronization, miniprobe headers and coding are removed. For example, the combination of 8-PSK and $9 \mathrm{kHz}$ channel gives user data rate of $14.4 \mathrm{kbits} / \mathrm{s}$ [10].

The modulation, coding and channel bandwidth are selected based on sensing results. As a consequence, the usable data rates of found usable HF channels characterize the data rate of the connection. This has its effects to how fast internet protocol (IP) packets (everything will be IP based on the future, right?) could be delivered. For example, assume that the channel conditions allow use of $24 \mathrm{kHz}$ channel with high modulation order (256-QAM) or only $9 \mathrm{kHz}$ with moderate modulation order (8-PSK). Then, delivery of 56 kbytes of IP-data would take $3.7 \mathrm{~s}$ or $31.1 \mathrm{~s}$, respectively. In this respect a particular interfering signal might appear as a moderately static signal or a variable signal depending of the selection of our PHY layer parameters.

Taking into account 8 bandwidths and 8 modulations we'll end up to 64 different combinations. Channel coding would increase the possibilities (or mess) even further. The highly adaptive PHY layer protocol in addition to measured channel statistics relation to it and the packet length of applications mean that the radio's cognitive decision making, i.e., cognitive engine (CE), should have "eyes in the back". This means that the CE should be very aware of the characteristics of the application layer data services with respect to possible PHY layer services.

In summary, the relevant signal characteristics are those that do predict the spectrum's future behavior with respect to the HF system. On the other hand, system's spectrum sensing performance should be able to distinguish the signals both in time and frequency domains. The time span, as discussed above, depends greatly of the system at hand. Especially in HF when the data rates are sufficiently low, i.e., transmissions lasts long, even the Moderately static signals may appear as a Variable signals - just to illustrate the problem

With a capable spectrum sensing module cognitive HF systems have a full potential to cognitively adapt to the HF channel, and access the spectrum dynamically, i.e., use dynamic spectrum access strategies in channel selection.

\section{Dynamic Spectrum Access}

First, some examples of a real HF channel measurement over a long ionospheric channel are shown as well as some examples of the local interference environment. After that the examples are discussed from a viewpoint of DSA on HF communications.

Fig. 1 shows channel measurement results over a HF ionospheric channel. The $\mathrm{X}$-axis presents $1 \mathrm{kHz}$ frequency slides over total of $50 \mathrm{kHz}$ frequency span, whereas the $\mathrm{Y}$-axis gives the time scale in minutes $(0-70)$. The darker the red color is the better the measured signal to noise ratio (SNR) has been. The sounding signal has been $1 \mathrm{kHz}$ BPSK modulated signal. The TX radio has been located on an amateur radio site at Azores island on Atlantic Ocean (station CR2X) and the RX radio has been at the Arkala amateur radio station in Finland (station OH8X) [11]. The distance between these locations is approximately $4500 \mathrm{~km}$.

Two main observations could be made. First, there are several $5-10 \mathrm{kHz}$ wide continuous spectrum "holes" present over the whole measurement interval. Secondly, none of these "holes" are present on the same frequency location over the whole 65 minutes measurement period.

Fig. 2 presents a channel interference measurement at 14 $\mathrm{MHz}$ over approximately $200 \mathrm{kHz}$ channel. X-axis shows frequency span $(0-200 \mathrm{kHz}), \mathrm{Y}$-axis is magnitude (logarithmic scale $-2-8)$ and $Z$-axis is time $(0-40 \mathrm{~s})$. The antenna has been located on the roof of an office building in city of Oulu, Finland.

Fig. 3 presents the same measurement than in Fig. 2, but the spectrum magnitudes being averaged over the whole $40 \mathrm{~s}$ measurement. This highlights the spectrum holes better.

The spectrum in Figs. 2 and 3 has had some very strong interferers that are also quite static. Most likely the three strongest signals had been BC-signals, and thus, are categorized to Static Signals class. In addition to that we observe some fading on interference seen as variations in signal magnitudes in Fig. 2. This indicates that those signals are received via an ionospheric channel rather than via a ground wave channel.

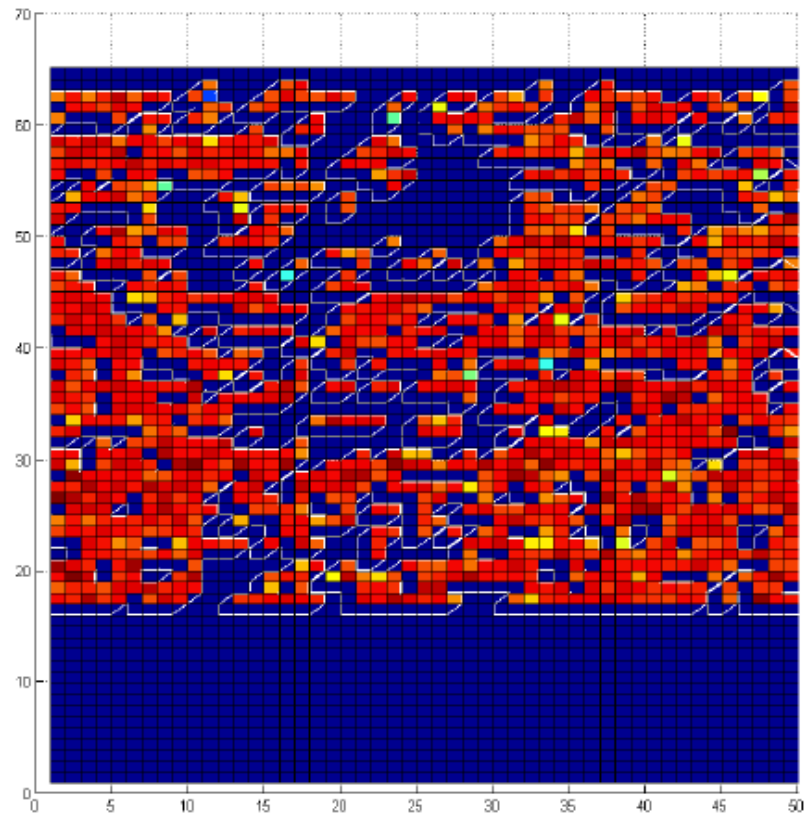

Figure 1. Propagation measurement over ionospheric channel. 


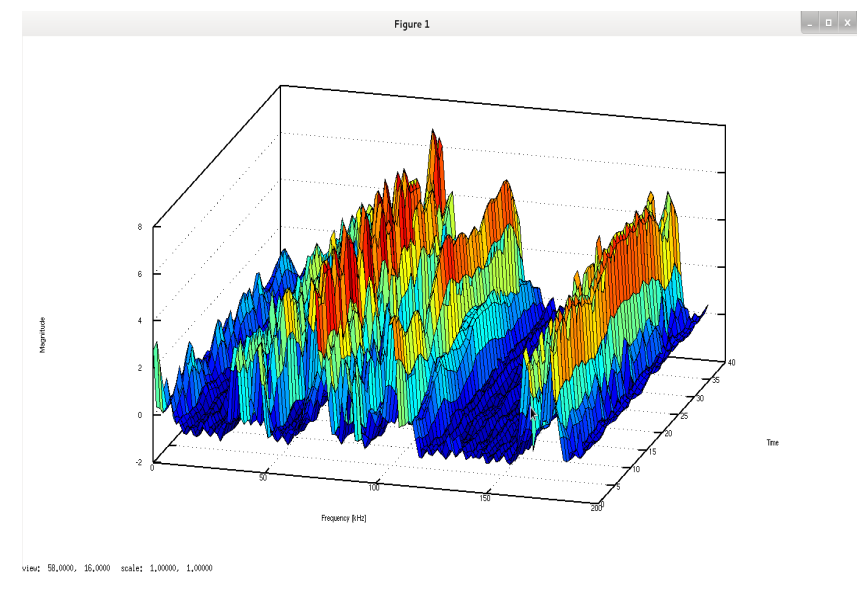

Figure 2. Measurement of local interferences as a function of time.

Even though this measurement was deliberately made in the most occupied part of the HF spectrum there are still quite much spectrum resources to use. It can be seen from Fig. 3 that there is at least $80 \mathrm{kHz}$ of unoccupied spectrum, the widest continuous part being almost $45 \mathrm{kHz}$ wide.

Based on these results the DSA has definitely its place: the spectrum offers the resources but not in any case in a static manner. Same kind of conclusions could be made from numerous High Frequency Industry Association (HFIA) presentations [12]. The old-fashioned HF data frequency planning combined with very limited frequency agile ALE protocols simply cannot exploit the spectrum holes in a dynamic manner. Therefore, new ALE protocols are being investigated, often called 4G ALE or WBALE (Wide band ALE) [13].

It is obvious based on the showed results that there are several possibilities to $\mathrm{HF}$ communications with varying bandwidth and duration. Naturally, since also channel quality varies different modulation and coding options should be used as well. This means that future cognitive HF systems should be aware of these possibilities and utilize them. The state-of-theart ALE protocol is, in any case, only an initial tool to enable the DSA and, of all things, the cognitivity in HF. More about these things are discussed in the next chapters.

\section{The Playground}

Like the previous chapters have brought forward the playground in HF spectrum is very complex with respect to optimally exploiting its full potential. First of all, the channel properties are under continuous change due to basic physics of the ionosphere. This has a consequence that both the propagation properties and the interference environment are far from static but are changing data packet by data packet basis at its worst.

However, there are presumably a lot of spectrum holes present in the HF spectrum all the time, but their location and properties are in constant change. The most state-of-the-art PHY layer protocol MIL-STD-188-110C, on the other hand, has defined a large scale of waveforms for different types of these spectrum holes.

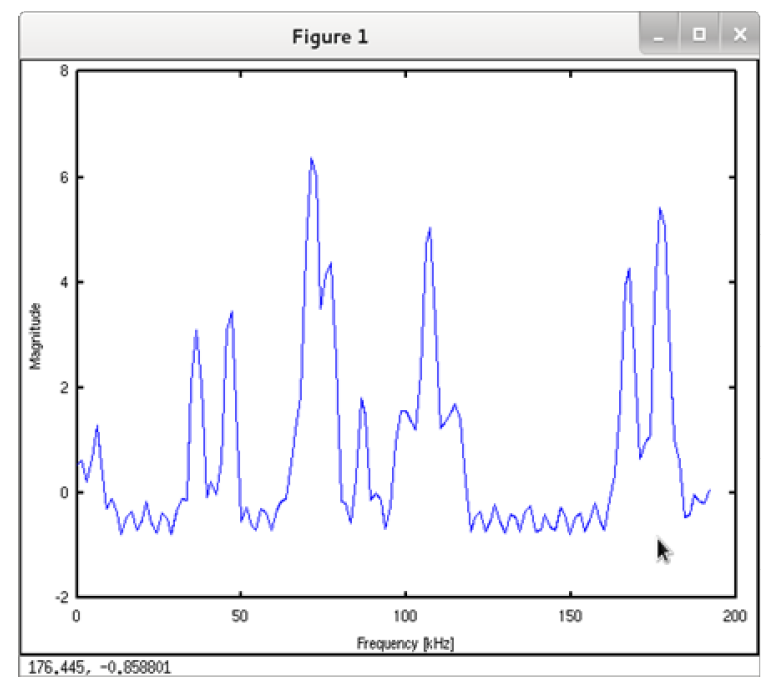

Figure 3. Time average of local interferences.

Thus, two basic conclusions could be drawn herein. Firstly, the spectrum holes should be found almost in real time just before the actual packet transmission takes place. Secondly, it would be advantageous if the PHY layer is flexible and its parameters could be adapted into current situation.

To meet these conditions in addition to an efficient spectrum estimator a cognitive HF radio needs an efficient medium access control (MAC) protocol as well. The wellknown request to send - clear to send (RTS-CTS) handshaking could be used the basic principle being that the communication channel is negotiated not until this point of the procedure. The authors recommend looking at numerous wireless sensor network and / or ad hoc MAC protocols found in literature.

The most difficult issue is the control channeling in HF, i.e., basically how to replace $2 \mathrm{G} / 3 \mathrm{G}$ ALE functionality with a protocol that would reduce the handshaking time from tens of seconds level to a sub-second level [14]. The other control channel related issue is how to raise the total number of the usable control channels far above the current number of channels in $2 \mathrm{G}$ and $3 \mathrm{G}$ ALEs. There is no plain answer to give yet, but only to endorse its attraction from the research point of view.

The limitations of the $2 \mathrm{G} / 3 \mathrm{G}$ ALE protocols are farreaching since, basically, their slow link set up times override the high data rate modes of MIL-STD-188-110C. This is easy to comprehend after taking into account the link establishment time when calculating the average data rate of the link. For example, a $120 \mathrm{kbits} / \mathrm{s}$ link and $56 \mathrm{kB}$ payload together with 10 $\mathrm{s}$ of link establishment time yield an average $13.7 \mathrm{kbits} / \mathrm{s}$ data rate that is only one tenth of the theoretical maximum. The ratio continues to deteriorate when multiple link establishments are needed per data transfer as might well occur in HF communications. 


\section{THE PLAYING RULES}

Due to "chaotic" number of actors and their "manners" in the HF band the behavior rules are hard to define. Of course, there are international and national frequency allocations on HF also, but the signal propagation does not obey the borders. One way to see the situation is to consider all the world's wifi radios on the same auditorium.

Bearing that in mind, a robust starting point would be to assume that our own link could be broken at any time. Once found frequency location is good only as long as the good luck continues - in interference sense. The propagation characteristics are easier to predict like stated earlier in this paper. So, it is evident that a new link should be set up as fast as possible after the previous had collapsed.

Assuming our link establishment protocol can establish a link at a sub-second rate, and thus, its effect on the overall data rate is acceptable, we'll still have some tricky questions to answer. As it is well-known, the higher the modulation order, the higher the SNR requirement. Furthermore, the higher the bandwidth of the signal, the higher the probability that an interferer will hit the signal is. Therefore, it can be said that "the higher the data rate the vulnerable the data transfer".

All these points are leading to multi dimensional problem field where basically all the factors, from the link establishment time and application requirements to current state of the spectrum (i.e., year, date, time) should be taken into account when designing the cognitive decision algorithms for $\mathrm{HF}$ communications.

Like stated before, the HF band is mainly considered for the long distance communications. However, it could be used also for shorter distances; the ground wave propagation could be used for distances up to few hundred kilometers. Since ground waves exist on all frequencies, not only those reflected from ionosphere, this has two benefits. First, in short distance HF communications the far reaching sky wave interference could be avoided by selecting the ground wave frequencies below the lowest usable frequency (LUF) or above the maximum usable frequency (MUF). This means that there are good possibilities to have a good channel for short links. Second, if some traffic can be transmitted through ground waves it reduces pressure to sky waves. This means reduced interference in sky waves. Naturally, since the data rates the HF communications offers are quite modest compared with many alternatives; these short range HF links could be used with applications that tolerate this data rate limit. Anyway, short range HF communications and its use for cognitive radio systems is a quite little studied area and, therefore, calls for research.

\section{FRIEND OR FOE?}

Let's say a foe. There are friends also, but the foe is a smarter guess. The foes are all the dull interfering emitters; the distant HF radios whose signals come and go (on the frequencies granted for our system). The friend is harder to define, excluding the members of own network, since a well behaving distant HF radio appear on our signal at any time due to ionospheric propagation, is turning from a friendly user to a foe.
The most fundamental question, if looking this aspect only from the technical point of view, is: if most of the actors in the HF band are not (from our perspective) obeying any rules, why should us? Thus, a smart and simple strategy would be to be nice for the members of the own HF network (obvious friends), and to dodge the strong foe interferers, no matter who they are, but not necessarily to pay any attention towards the weak foe signals.

The above statement has to be understood in the right context. It is not intended that frequency regulations are violated or that interference is caused to other systems operating at the same frequencies. Frequency regulations obviously are based on clear priorities and fixed allocations while cognitive radio systems require the widest possible flexibility for the best overall performance.

\section{CONCLUSIONS}

This paper has discussed some of the very fundamentals of the HF band data transmissions from the view point of cognitive radios. Based on our own initial trial measurements it is evident that even long distance links could support tens of $\mathrm{kHz}$ links. Same kind of results can be found from numerous HFIA presentations. Based on the own measurements it was shown that local interference environment could have wide variance in the interfering signals, and at the same time, offer tens of $\mathrm{kHz}$ spectrum holes.

It was pointed out that in addition to an efficient spectrum sensing, an efficient MAC protocol, and first and foremost, an efficient link establishment protocol is needed to constitute the cognitive HF radio and networking. With the help of those tools the cognitive engine has multidimensional problem field to solve in order to exploit the resources of the HF band.

After all, the arguments above only emphasize the possibilities for cognitive networking on HF. The more there are decisions to make the more possibilities there are, is one way to see the HF playground.

\section{REFERENCES}

[1] International Telecommunication Union, Handbook Frequency-adaptive communication systems and networks in the MF/HF bands

[2] Electronics Technician Volume 7-Antennas and Wave Propagation NAVEDTRA 14092

[3] MIL-STD-188-141A

[4] MIL-STD-188-141B

[5] Report ITU-R SM.2152, 2009

[6] WEWN Vandiver, AL HF broadcast station

[7] M. Edirisinghe and V. Cooray, "Fine Structure Signatures in Ground Flashes as a Source for HF Radiation", International Letter of Chemistry, Physics and Astronomy, 2014

[8] MIL-STD-188-110C

[9] E. Johnson "Revision of US Military HF Radio Standards", HFIA meeting, San Diego, 2012

[10] E. Johnson, W. Furman, M. Jorgenson, J. Nieto and R. Blocksome "Wideband HF Waveform Design for MIL-ST-188-110C", HFIA meeting, San Diego, 2010

[11] http://www.radioarcala.com

[12] http://www.hfindustry.com/meeting_reviews.htm 
[13] E.E. Johnson, E. Koski, W.N. Furman, M. Jorgenson and J. Nieto, "Third-generation and wideband HF radio communications", Artech House, Norwood MA, 2013
[14] W. Furman, E. Koski, "Next Generation ALE Concepts", HFIA meeting, San Diego, 2009 